Rosel Mieko Yamamoto Nomura'

SEIZO MIYADAHIRA ${ }^{2}$

MARCELO ZUGAiB ${ }^{3}$

\title{
Avaliação da vitalidade fetal anteparto
}

\author{
Antenatal fetal surveillance
}

Revisão

Palavras-chave

Frequência cardíaca feta

Hipóxia fetal

Monitorização fetal

Ultra-sonografia

Ecocardiografia doppler

Sofrimento fetal

Keywords

Heart rate, fetal

Fetal hypoxia

Fetal monitoring

Ultrasonography

Echocardiography, doppler

Fetal distress

Correspondência:

Roseli Mieko Yamamoto Nomur Departamento de Obstetrícia e Ginecologia da Faculdade de Medicina da Universidade de São Paulo Avenida Dr. Enéas de Carvalho Aguiar, $255-10^{\circ}$ andar, sala 10.037 (EP $05403-000$ - São Paulo (SP), Brasil Fone: (11) 3069-6209 - Fax: (11) 3069-8183 E-mail: roseli.nomura@terra.com.br

Recebido

20/8/09

Aceito com modificações

\section{Resumo}

C contexto atual da atividade médica exige do obstetra e ginecologista ampla compreensão dos avanços científicos e tecnológicos de sua área. $\bigcirc$ objetivo primordial da avaliação fetal antenatal é identificar fetos de risco para eventos adversos ou para o óbito e, assim, atuar preventivamente para evitar o insucesso. $\bigcirc$ perfil biofísico fetal atinge sua máxima eficiência quando aplicado dentro do contexto clínico de cada caso. Em gestações de alto risco, a doplervelocimetria da artéria umbilical mostrou-se útil para melhorar os resultados perinatais. Na restrição de crescimento fetal por insuficiência placentária grave, antes da $34^{a}$ semana de gestação, a doplervelocimetria do ducto venoso tem sido importante instrumento na condução dos casos. Nenhum teste isoladamente é considerado o melhor na avaliação da vitalidade fetal anteparto, entretanto, a análise conjunta de todos os métodos irá propiciar melhor compreensão da resposta fetal à hipóxia.

\section{Abstract}

The present context of medical practice demands from the obstetrician and gynecologist broad understanding of the scientific and technological advances of the area. The main purpose of prenatal evaluation is to identify fetuses at risk for adverse events or death, for preventive action to avoid mishappenings. The determination of fetal biophysical profile reaches its maximum efficiency when applied within the clinical context of each case. In high risk gestations, the Doppler velocimetry of the umbilical artery has shown to be useful to improve perinatal outcome. In the fetal growth deficit, due to severe placentary insufficiency, Doppler velocimetry of the venous duct has been showing to be an important tool in handling of the cases before the $34^{\text {th }}$ week of gestation. Although no test itself is considered the best to evaluate the fetus's prenatal vitality, the joint analysis of all methods may lead to a better understanding of the fetal response to hypoxia.
Disciplina de Obstetrícia do Departamento de Obstetrícia e Ginecologia da Faculdade de Medicina da Universidade de São Paulo - USP - São Paulo (SP), Brasil.

Professor do Departamento de Obstetrícia e Ginecologia da Faculdade de Medicina da Universidade de São Paulo - USP - São Paulo (SP), Brasil.

2 Professor Titular do Departamento de Obstetrícia e Ginecologia da Faculdade de Medicina da Universidade de São Paulo - USP São Paulo (SP), Brasil.

${ }_{3}^{3}$ Professor Titular do Departamento de Obstetrícia e Ginecologia da Faculdade de Medicina da Universidade de São Paulo - USP São Paulo (SP), Brasil. 


\section{Introdução}

O objetivo primordial da avaliação fetal antenatal é identificar fetos de risco para eventos adversos ou para o óbito e, assim, atuar preventivamente para evitar o insucesso. Apesar do vasto emprego da alta tecnologia na propedêutica fetal, são poucas as evidências norteando a aplicação apropriada dos métodos disponíveis, ou que demonstrem a efetividade dos exames em melhorar os resultados perinatais. No entanto, nas gestações de alto risco, a propedêutica da vitalidade fetal anteparto é empregada de forma rotineira na prática obstétrica, geralmente com o intuito de identificar fetos que se beneficiam de intervenções oportunas, ou por razões de exposição profissional, a responsabilidades que possam vir ser questionadas no futuro.

Atualmente, a avaliação da vitalidade fetal anteparto é solicitada para as gestações nas quais o risco de comprometimento fetal é sabidamente aumentado. São gestantes portadoras de intercorrências clínicas e/ou obstétricas, que potencialmente se relacionam à disfunção placentária ou a patologias fetais, e expõem o concepto ao risco de sequelas a longo prazo ou ao óbito. Os testes utilizados baseiam-se na premissa de que o feto com distúrbios de oxigenação apresentará quadro clínico caracterizado por respostas adaptativas fisiológicas, que podem evoluir para sinais de descompensação fetal.

Uma das respostas adaptativas do feto à hipoxemia é o mecanismo hemodinâmico fetal de compensação, o qual envolve a estimulação do sistema nervoso autônomo fetal, provocando aumento da resistência vascular periférica e gradativo aumento da frequência cardíaca fetal (FCF). Ocorre direcionamento de maior proporção do fluxo sanguíneo proveniente da placenta, rico em oxigênio e nutrientes, para o cérebro, o coração e adrenais e subsequente redução da perfusão renal, do trato gastrintestinal e o restante do corpo. Nesse processo, o ducto venoso desempenha papel fundamental. Na resposta inicial à hipoxemia fetal, maior proporção de fluxo é direcionada a esse, em detrimento do fluxo ao seio portal, reduzindo o suprimento ao fígado fetal. Nessas fases iniciais de hipoxemia ocorre também aumento do fluxo ao cérebro fetal, por mecanismos de autorregulação da circulação, o que é denominado centralização da circulação fetal.

Outros órgãos passam a ter seu funcionamento depreciado no processo adaptativo. A perfusão renal prejudicada propicia redução no volume de líquido amniótico (vLA) pela menor diurese fetal. A movimentação fetal se reduz, pois é um indicador indireto da integridade e do funcionamento do sistema nervoso central. Na hipoxemia aguda, a movimentação fetal diminui como mecanismo de conservação da energia pelo feto. A estimulação de quimiorreceptores provoca respostas mediadas pelo vago, as quais reduzem a FCF e podem se manifestar clinicamente pelo aparecimento de desacelerações tardias. Mudanças significativas são observadas na circulação e nos parâmetros biofísicos do feto, com desenvolvimento de hipóxia e acidemia, aumentando o risco de lesão de órgãos e desenvolvimento de sequelas. Diversos estudos analisam a sequência de alterações nos diversos parâmetros avaliados na propedêutica fetal. Apesar de existirem variações descritas, em geral, na insuficiência placentária e hipoxemia fetal progressiva, anormalidades na FCF e no fluxo sanguíneo da artéria umbilical são frequentemente consideradas sinais precoces do comprometimento fetal. Posteriormente, são verificadas alterações no fluxo de outros vasos fetais, seguidas de anormalidades nos parâmetros agudos do perfil biofísico fetal, PBF (movimentos respiratórios, corpóreos e tônus). Nem todos os fetos comprometidos demonstram todas as alterações descritas e, ainda assim, poderão apresentar acidemia no nascimento. Portanto, nenhum teste isoladamente é considerado o melhor na avaliação da vitalidade fetal anteparto, mas sim a análise conjunta de todos os métodos.

\section{Métodos de avaliação fetal antenatal}

Registro de movimentos fetais

A investigação sobre os movimentos fetais tem grande importância na prática obstétrica. Eles são percebidos pela mãe no segundo trimestre gestacional, entre a $17^{\mathrm{a}} \mathrm{e}$ a $20^{a}$ semana de gestação. É dado de fácil observação que pode ser investigado na anamnese bem dirigida. A movimentação fetal se reduz frente à hipoxemia. Quando são relatadas mudanças abruptas no padrão de movimentação do concepto, elas podem revelar comprometimento fetal, exigindo investigação por propedêutica especializada.

Não existe padronização quanto aos métodos de registro dos movimentos fetais. Apesar do Ministério da Saúde ${ }^{1}$ recomendar, para as gestações de baixo risco, o registro diário de seis movimentos fetais em uma hora de observação a partir da $34^{a}$ semana, não existem evidências claras do limite abaixo do qual ocorra maior risco de comprometimento fetal. Um estudo internacional composto por 68.654 casos não demonstrou diferença significativa na prevenção de mortes fetais tardias, potencialmente evitáveis, entre gestantes instruídas a realizar contagem rotineira dos movimentos fetais em relação ao Grupo Controle ${ }^{2}$. Ademais, revisão sistemática conclui serem insuficientes as evidências para se recomendar a avaliação rotineira da contagem de movimentos fetais na prevenção do óbito fetal ${ }^{3}$.

Portanto, não existe definição sobre o número ideal de movimentos fetais, nem da duração para fins de contagem. A reprodutibilidade e a predição desses métodos tornam-se precárias e o dado qualitativo parece ter melhor 
aplicabilidade na prática clínica diária. Um estudo recente demonstra que informações escritas oferecidas às gestantes sobre a importância da redução da movimentação fetal, e o estímulo ao controle deste parâmetro promoveram melhor conduta e estão associados a menor ocorrência de óbito fetal ${ }^{4}$.

\section{Cardiotocografia anteparto de repouso}

A cardiotocografia é método frequentemente empregado para a avaliação do bem-estar fetal, principalmente no seguimento das gestações de alto risco. Também conhecida como monitorização fetal eletrônica, baseia-se na análise de registros gráficos da FCF e do tônus uterino. As alterações nos traçados são observadas em diversos estados comportamentais do feto (ciclo sono-vigília), nos distúrbios de sua oxigenação ou em situações decorrentes da utilização de medicações pela gestante. Apesar de não haver evidências de estudos aleatorizados que comprovem a redução do risco de óbito fetal com o uso do método 5 , a cardiotocografia está presente em todos os centros de referência que acompanham gestações de risco, tornando-se parte integrante da assistência pré-natal especializada.

A cardiotocografia de repouso é a modalidade mais utilizada no período anteparto, por ser de fácil uso e sem risco adicional à saúde materna ou fetal. O método é melhor utilizado a partir do momento em que a maturidade do sistema nervoso autônomo fetal propicia a regulação da FCF, isto é, ao final do segundo trimestre de gestação. A imaturidade fetal prejudica a interpretação dos traçados. A influência do sistema parassimpático sobre a FCF aumenta gradativamente com o avanço da idade gestacional. A interação dos sistemas, simpático e parassimpático, estabelece a variabilidade da FCF, parâmetro fundamental na interpretação do traçado cardiotocográfico.

A interpretação visual do traçado exige a adoção de critérios bem estabelecidos. O quadro 1 contém as definições estabelecidas pelo consenso internacional para interpretação visual, recentemente revisado em $2008^{6}$. Apesar dessas definições serem direcionadas para o período intraparto, são aplicáveis também para o anteparto. A presença de acelerações transitórias da FCF (duas durante 20 minutos) com amplitude superior a 15 batimentos por minuto (bpm) por 15 segundos ou mais, indica bemestar fetal ${ }^{7}$. Um critério diverso tem sido aplicado para o período anterior à $32^{\mathrm{a}}$ semana, quando são caracterizadas como transitórias as acelerações superiores a dez bpm por dez segundos ou mais ${ }^{8}$.

Em geral, a presença de acelerações e a variabilidade normal da FCF são achados que indicam a normoxemia fetal ${ }^{9}$. As desacelerações da FCF no anteparto, por sua vez, são indicativas de anormalidades. As desacelerações tardias são mediadas pela estimulação de quimiorreceptores nas situações de hipoxemia fetal ${ }^{10}$. Desacelerações prolongadas mais frequentemente associam-se ao comprometimento fetal ${ }^{11}$. A elevação da FCF basal e a redução de variabilidade são sinais adicionais de acidose metabólica fetal ${ }^{12}$.

Em nosso meio, habitualmente são utilizados o índice cardiotocométrico de Zugaib e Behle ${ }^{13}$, modificados para a interpretação dos traçados. Esta forma de interpretação é didática ao impor disciplina na análise de todos os parâmetros da FCF. Quando normal, cada parâmetro recebe pontuação específica: linha de base entre 110 e 160 bpm (1 ponto); variabilidade entre 10 e $25 \mathrm{bpm}$ (1 ponto); presença de pelo menos uma aceleração transitória (2 pontos); e ausência de desacelerações (1 ponto). $\mathrm{O}$ índice cardiotocométrico é constituído pela somatória dos pontos obtidos, classificando o feto em: Ativo, quando tem

Quadro 1 - Interpretação dos parâmetros da FCF avaliados pela cardiotocografia

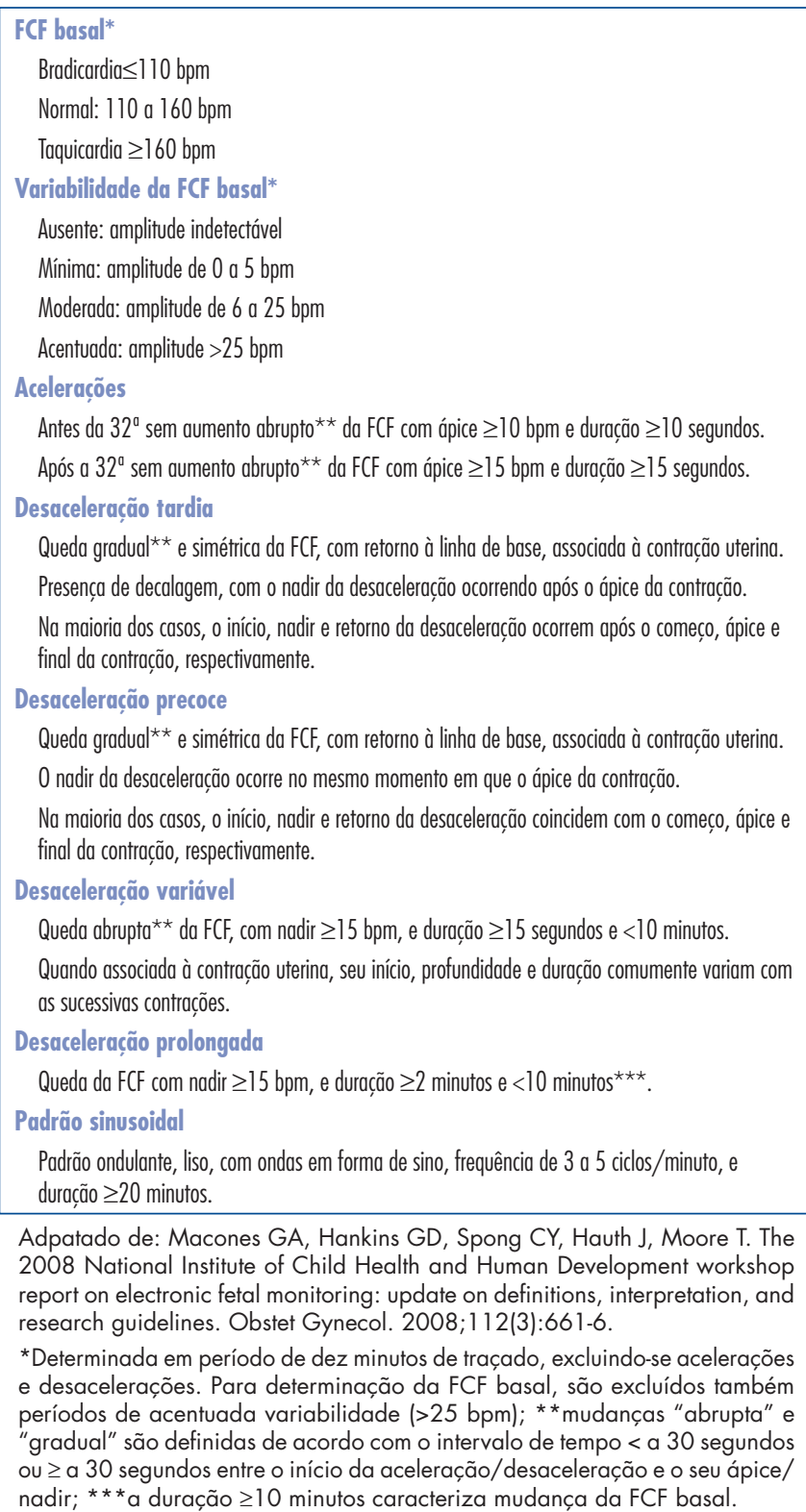


pontuação 4 e 5 (normal); Hipoativo, com pontuação 2 e 3 (suspeito); e Inativo, com pontuação 0 e 1 (alterado).

Os fetos classificados como hipoativos podem ter como causa associada o sofrimento fetal, o período de sono fisiológico e o uso de drogas (sedativos, betabloqueadores). É recomendada a complementação da propedêutica fetal por outras modalidades cardiotocográficas complementares, como o teste da estimulação sônica ou o perfil biofísico fetal. O padrão cardiotocográfico terminal é caracterizado quando o feto apresenta-se inativo, com variabilidade mínima ou ausente e presença de desacelerações recorrentes. Eventualmente, também são observadas alterações na linha de base com taquicardia ou bradicardia fetal.

\section{Cardiotocografia estimulada}

Este método objetiva primordialmente modificar o estado de sono fetal para o de vigília. Trata-se, portanto, de método complementar quando a cardiotocografia de repouso apresenta resultado suspeito. É de grande utilidade para reduzir a proporção de exames falso-positivos da cardiotocog rafia anteparto de repouso. A estimulação fetal pode ser vibroacústica (estímulo sônico), mecânica (movimentação do polo cefálico) ou vibratória. Apesar da metanálise não demonstrar benefícios da manipulação mecânica do feto para reduzir a incidência de traçados anormais na cardiotocografia ${ }^{14}$, um estudo aleatorizado constata que a estimulação manual do feto reduz significativamente o tempo necessário para se atingir padrões normais na cardiotocografia ${ }^{15}$.

O teste da estimulação sônica é padronizado pela utilização de fonte sonora com frequência de 500 a 1.000 $\mathrm{Hz}$ e pressão sonora de 110 a $120 \mathrm{~dB}$. Após efetuar traçado cardiotocográfico basal, com no mínimo dez minutos de duração, e caracterizado estado de hipoatividade ou inatividade fetal, aplica-se o estímulo por três segundos, adaptando-se a fonte sonora sobre o abdome materno na região correspondente ao polo cefálico.

Foram verificados dois tipos de respostas: a motora e a cardíaca, ambas mediadas por via neural. Os fetos hígidos apresentam invariavelmente movimentos corpóreos amplos e rápidos. Ademais, nota-se também, incremento na FCF que se mantém por algum tempo, fruto da liberação de catecolaminas. Em metanálise envolvendo nove estudos com total de 4.838 participantes, foi concluído que a estimulação sônica reduz a incidência de exames não reativos $(\mathrm{R} R=0,62$; IC95\% $=0,5-0,7)$ e reduz a média de tempo de exame (- 9,9 minutos, IC95\%=-9,37 --10,50 minutos) ${ }^{16}$.

De acordo com a resposta cardíaca fetal, o feto pode ser classificado em: reativo, quando há resposta cardíaca com amplitude de pelo menos $20 \mathrm{bpm}$ (pico) e duração de pelo menos três minutos; hiporreativo quando a amplitude for menor que $20 \mathrm{bpm}$ e/ou duração for menor que três minutos; e, não reativo, quando não se verifica resposta cardíaca fetal. Classifica-se, ainda, como resposta bifásica quando após o término da resposta aparecem acelerações transitórias, e resposta monofásica quando isso não ocorre. É considerado normal o feto reativo ou quando há resposta bifásica.

\section{Cardiotocografia com sobrecarga}

O teste de sobrecarga mais utilizado é o teste de Pose, que consiste em induzir contrações uterinas pela infusão de ocitocina e avaliar a resposta da FCF. Passou a ser pouco utilizada a partir da introdução rotineira de outros métodos de avaliação da vitalidade fetal, principalmente a doplervelocimetria ${ }^{17}$. Os resultados anormais nesse teste são indicativos de resolução da gestação, porém sua análise deve ser feita de forma cuidadosa, considerando-se o quadro clínico materno e fetal. Alguns estudos sugerem boa predição para resultados adversos ${ }^{18}$, mas outros não demonstram qualquer progresso na predição da morbidade perinatal, mesmo quando seu uso é associado à cardiotocografia de repouso ${ }^{19}$.

\section{Cardiotocografia computadorizada}

Inicialmente a cardiotocografia computadorizada teve como base um banco de dados com 8.000 cardiotocografias de repouso. A nova versão, de 1994, é atualizada com análise de 48.339 registros da FCF (Sonicaid 8002). Outros programas surgiram posteriormente utilizando sistemas computadorizados ${ }^{20}$.

Os critérios de normalidade do sistema 8002 são conhecidos como critérios de Dawes/Redman. São analisados os seguintes parâmetros: movimentos fetais por hora, calculado com base na percepção materna por marcador de eventos; FCF basal (normal de 116 a 160 bpm), calculada com base na divisão do traçado em 16 episódios de 3,75 segundos, nos quais é mensurada a média da frequência; número de acelerações da FCF, de amplitude superior a 10 e a 15 bpm; episódios de alta e de baixa variações da FCF, em minutos, identificados pela variação pico a pico da frequência a partir da linha de base, em períodos de cinco a seis minutos consecutivos; variação de curto prazo, calculada pela média das diferenças sucessivas dos intervalos de pulso pelo qual é calculada a variação média de cada período (em ms $)^{21,22}$.

Pelo menos um episódio de alta variação deve estar presente a partir da $28^{a}$ semana em fetos normais. Os episódios de baixa variação podem durar até 50 minutos em fetos saudáveis próximo ao termo da gestação. Por este motivo não existe período preestabelecido para a duração do exame, esta irá depender do preenchimento dos critérios, no mínimo 10 minutos e no máximo, 60 . $\mathrm{Na}$ ausência de episódios de alta variação, a variação de curto prazo deve ser considerada como melhor guia para análise do resultado ${ }^{23}$. 
As alterações detectadas pelo sistema associam-se significativamente a resultados perinatais adversos ${ }^{24,25}$, e quando os critérios não são atingidos, caracteriza-se situação de risco para o comprometimento da vitalidade fetal, sendo indicada complementação da propedêutica por meio de outros métodos. Em 71 gestações de alto risco, avaliadas pela cardiotocografia computadorizada até sete dias antes do parto, maiores taxas de morbidade neonatal imediata estão associadas aos resultados anormais ${ }^{26}$.

O método tem mostrado particular utilidade nos estudos que investigam aspectos da fisiologia fetal. Em gestações de termo complicadas pelo diabetes, a glicemia materna no momento do exame apresenta significativa correlação positiva com a FCF basal, bem como significativa correlação negativa com a variação de curto prazo $^{27}$.

\section{PBF}

O PBF é método da propedêutica do bem-estar fetal que estuda conjuntamente as atividades biofísicas e o vLA. Fundamenta-se na hipótese de que as variáveis biofísicas fetais (movimentos respiratórios, movimentos corpóreos, tônus fetal e resultados da cardiotocografia) refletem a integridade funcional do sistema nervoso central e, como tal, espelham o estado de oxigenação. Cada parâmetro recebe pontuação 2 , de acordo com critérios específicos observados em um período máximo de 30 minutos: resultado da cardiotocografia demonstrando acelerações transitórias; um ou mais episódios de movimentos respiratórios rítmicos com 30 segundos de duração; três ou mais movimentos corpóreos discretos ou um amplo; tônus fetal presente com extensão de extremidade ou da coluna com retorno à posição de flexão; bolsão de líquido amniótico com diâmetro vertical superior ou igual a $2 \mathrm{~cm}^{28}$, ou, segundo alguns autores, índice de líquido amniótico (ILA) superior ou igual a $5,0 \mathrm{~cm}^{29}$. Alguns autores justificam o uso do critério do maior bolsão por sua maior especificidade quando comparado ao ILA, o que reduz a probabilidade de intervenções por anormalidade deste parâmetro ${ }^{30}$. A somatória corresponde ao PBF e os fetos comprometidos apresentam alterações progressivas nestes parâmetros.

A interpretação do escore final do PBF segue o seguinte $^{28}$ : 10/10 ou 8/10 (vLA normal), risco de asfixia fetal de 1/1.000 dentro de uma semana, se não houver intervenção; 8/10 (vLA anormal), risco de asfixia fetal de 89/1.000 dentro de uma semana, se não houver intervenção; 6/10 (vLA normal), teste suspeito, possível asfixia fetal, repetir o teste dentro de 24 horas ou indicar interrupção da gestação; 6/10 (vLA anormal), risco de asfixia fetal de 89/1.000 dentro de uma semana, se não houver intervenção; 0 a 4/10, risco de asfixia fetal de 91 a 600/1.000 dentro de uma semana, se não houver intervenção.
A relação entre a acidemia fetal e os resultados do PBF é demonstrada em estudos clínicos ${ }^{31,32}$. A mortalidade e morbidade perinatal também se relacionam com os resultados deste teste ${ }^{33}$. Quanto às sequelas a longo prazo, existe correlação inversa entre a incidência de paralisia cerebral e o último resultado do $\mathrm{PBF}^{34}$. Resultados neurológicos adversos e retardo mental são reduzidos de forma significativa no seguimento dos recém-nascidos de gestações de alto risco conduzidas de acordo com resultados do PBF, quando comparadas com pacientes de baixo risco não testadas. Estudos observacionais demonstram que o uso do PBF está associado à redução de 61 a 76\% na mortalidade perinatal corrigida, quando comparado a controles históricos ${ }^{35}$.

O PBF atinge sua máxima eficácia quando utilizado dentro do contexto clínico de cada caso. O teste apresenta falso-negativo para óbito fetal de $0,07 \%{ }^{36,37} \mathrm{e}$ falso-negativo para resultados perinatais adversos (óbito perinatal, cesárea por sofrimento fetal, Apgar no quinto minuto inferior a sete, convulsões neonatais ou hemorragia intracraniana) de 5,1\% ${ }^{38}$.

Existe controvérsia sobre os efeitos da corioamnionite subclínica nos resultados do PBF. Estudos não consideram este método um teste sensível para detecção de infecção intraútero ${ }^{39}$. Não foi demonstrada relação entre sinais histológicos de inflamação aguda da placenta e os resultados do PBF ou de seus componentes individuais, nos casos de rotura prematura de membranas antes da $32^{a}$ semana $^{40}$. Além disto, dados analisados em revisão sistemática não permitiram concluir que a monitoração dos movimentos respiratórios fetais fosse adequada para predizer o trabalho de parto prematuro ${ }^{41}$.

\section{Doplervelocimetria}

A aplicação pioneira da doplervelocimetria, em Obstetrícia, ocorreu em 1977, quando Fitzgerald e Drumm ${ }^{42}$ obtiveram registros sonográficos de vasos umbilicais, utilizando um dispositivo de registro contínuo do fluxo sanguíneo. Numerosas investigações, em diversos centros, consolidaram o exame ao demonstrarem a existência da associação clara entre os valores anormais com os resultados perinatais adversos. Na década de 1990, a doplervelocimetria foi incorporada definitivamente ao arsenal propedêutico obstétrico e passou a desempenhar papel fundamental no seguimento de gestações de alto risco, propiciando nova dimensão no campo da avaliação fetal.

Essa é um método que efetua a mensuração das velocidades de fluxo em vasos da circulação materna e fetal. Fornece informações a respeito do fluxo uteroplacentário e da resposta circulatória fetal a eventos fisiológicos e patológicos. Com o mapeamento colorido é possível retratar padrões bidimensionais de fluxo sobrepostos à imagem 
das estruturas anatômicas. A análise de vasos específicos, pelas mudanças na frequência Doppler, de acordo com o ângulo de insonação, permite a obtenção da velocidade do sangue. Dessa forma, a doplervelocimetria propicia a investigação das alterações hemodinâmicas que caracterizam a condição fetal.

O estudo das velocidades de fluxo oferece informações sobre vários aspectos da circulação: presença e direção do fluxo, perfil da velocidade, volume de fluxo e resistência ao fluxo. Na prática obstétrica, os índices Doppler da artéria umbilical têm sido amplamente utilizados na avaliação da resistência no território placentário. Habitualmente são utilizados os seguintes índices doplervelocimétricos: relação sístole/diástole $(\mathrm{A} / \mathrm{B})$, que corresponde à razão entre o pico sistólico e a velocidade diastólica final; o índice de resistência (IR), o qual representa a divisão da diferença entre o pico sistólico e a velocidade diastólica final com o pico sistólico; e o índice de pulsatilidade (IP) que é a razão da diferença entre o pico sistólico e a velocidade diastólica final, com a velocidade média obtida pela análise do envelope da onda.

Alguns fatores alteram a análise pelo Doppler: a idade gestacional (existe declínio progressivo da relação A/B da artéria umbilical com o avanço da gestação); a FCF (as alterações nos índices não são significativas com a FCF entre 120 e 160 bpm $)^{43}$; os movimentos respiratórios fetais; os movimentos fetais; e o local onde se obtém a amostra (os índices do Doppler da artéria umbilical são menores nas proximidades da inserção do cordão na placenta, quando comparados ao cordão próximo à inserção abdominal) ${ }^{44}$. Não parece haver influência dos estados comportamentais nos resultados dos índices doplervelocimétricos da artéria umbilical, mas este não pode ser afirmado quanto às artérias cerebrais, havendo redução dos índices no estado $2 \mathrm{~F}$ (sono ativo) $)^{45}$.

A interpretação clínica das informações obtidas na análise das ondas de velocidade de fluxo é variável de acordo com cada vaso específico abordado.

\section{Artéria uterina}

A invasão trofoblástica inadequada, impedindo o pleno remodelamento das artérias espiraladas maternas, caracteriza a persistência da resistência elevada na circulação na artéria uterina e na presença de incisura. Essas alterações, verificadas entre a $22^{\mathrm{a}}$ e $24^{\mathrm{a}}$ semanas, associam-se à ocorrência posterior de pré-eclâmpsia, restrição de crescimento fetal e óbito perinatal ${ }^{46}$. Entretanto, a baixa sensibilidade do método ( $41 \%$ para predição da pré-eclâmpsia e $16 \%$ para a restrição de crescimento fetal ${ }^{47}$ prejudica a utilização do método na prática clínica, pois os resultados falso-positivos propiciam, por vezes, condutas equivocadas. Ademais, esses resultados não se referem à vitalidade fetal especificamente, e sim a patologias associadas com a má placentação. Portanto, não é considerado método de avaliação fetal.

\section{Artéria umbilical}

O desenvolvimento e o crescimento normal do feto caracterizam-se pelo fluxo diastólico de elevada velocidade nas artérias umbilicais. Em fetos com crescimento restrito, por sua vez, esse fluxo diastólico se reduz, podendo ser ausente ou mesmo reverso. A diminuição progressiva do fluxo diastólico nas artérias umbilicais representa avarias na vascularização dos vilos placentários. Estudos relatam que o aumento na relação $\mathrm{A} / \mathrm{B}$ está associado à obliteração das pequenas arteríolas musculares nos vilos terciários ${ }^{48}$. Esses achados sugerem que o desenvolvimento insuficiente desses vilos e da estrutura vascular fetoplacentária promove aumento na impedância, prejudicando a transferência de nutrientes e gases entre a placenta e o feto ${ }^{49}$. Em modelos experimentais, foi verificado que quando a obliteração atinge aproximadamente $30 \%$ do território placentário há aumento da relação $\mathrm{A} / \mathrm{B}$ da artéria umbilical, e quando 60 a $70 \%$ da vascularização está comprometida ocorre a diástole zero ou fluxo reverso ${ }^{50}$.

Complicações como a restrição de crescimento fetal e a pré-eclâmpsia, pela insuficiência placentária, resultam na deficiência crônica de nutrição e oxigenação fetal. Por sua vez, o feto desacelera seu ritmo de crescimento, reduz a movimentação, faz uso de mecanismos compensatórios pela centralização da sua circulação frente à hipóxia crônica. A elevação anormal dos índices doplervelocimétricos da artéria umbilical precede as alterações da FCF e do $\mathrm{PBF}^{51,52}$. Apesar da grande heterogeneidade, estudos aleatorizados controlados em gestações de alto risco demonstram a eficiência da doplervelocimetria da artéria umbilical em melhorar os resultados perinatais ${ }^{53-57}$. Entretanto, para gestações de baixo risco, o uso desta técnica da artéria umbilical como teste de rastreamento não demonstra benefício em relação aos resultados perinatais ${ }^{58,59}$.

A relação A/B superior a 3,0 ou o IR inferior a 0,6 demonstram habilidade em discriminar casos de maior risco após a $28^{a}$ semana de gravidez ${ }^{58}$. Além disso, os resultados obtidos na artéria umbilical podem ser comparados a curvas de normalidade, e considerados anormais os valores que se encontrem acima do percentil 95 para a idade gestacional em que é realizado o exame ${ }^{60}$.

Em fetos com crescimento restrito, a diástole zero ou reversa da artéria umbilical associa-se à hipóxia fetal ${ }^{61}$, maior morbidade e mortalidade perinatal ${ }^{62}$. Em casuística nacional ${ }^{62}$ de 170 casos de diástole zero ou reversa em casos sem anomalias fetais, a mortalidade fetal observada foi de $13,7 \%$ e a mortalidade neonatal de $22,1 \%$. A mortalidade em recém-nascidos com peso inferior a $1.000 \mathrm{~g}$ foi de $74,7 \%$, e quando a idade gestacional foi inferior a 31 semanas, de $66,3 \%$. A análise multivariada demonstrou 
que o peso do recém-nascido foi a melhor variável capaz de predizer o óbito perinatal ${ }^{62}$, aspecto esse também constatado em estudos internacionais ${ }^{63}$.

A frequência de diástole zero ou reversa na artéria umbilical é de $2 \%$ em gestações de alto risco, e de $0,3 \%$ nas gestações em geral. Uma vez detectada esta anormalidade, o fluxo diastólico pode apresentar melhora com o tempo, embora frequentemente seja apenas transitória. Dias ou semanas podem decorrer até que o feto demonstre evidências adicionais de comprometimento do seu estado de oxigenação, especialmente nas gestações longe do termo. A vigilância do bem-estar fetal é fundamental por meio de todos os métodos disponíveis. Pela gravidade do comprometimento fetal, a interrupção imediata da gestação é recomendada nos casos de diástole zero ou reversa, após a $34^{a}$ semana gestacional ${ }^{64-66}$. Com os recentes avanços da Neonatologia, o manejo expectante é reservado somente para as gestações distantes do termo.

$\mathrm{Na}$ restrição do crescimento fetal grave, a idade gestacional no nascimento inferior a 27 semanas e o peso do recém-nascido inferior a $922 \mathrm{~g}$ constituem os fatores com melhor predição para comprometimento do desenvolvimento neurológico após dois anos de vida ${ }^{67}$. Nessas situações, a conduta conservadora é adotada mediante vigilância diária do bem-estar fetal, pela propedêutica fetal disponível. A corticoterapia antenatal em gestações abaixo da $34^{\mathrm{a}}$ semana deve ser efetuada, com melhores efeitos quando a administração do corticoide inicia-se 48 horas antes do nascimento. A doplervelocimetria venosa é particularmente útil no manejo desses casos, auxiliando na identificação dos casos com disfunção cardíaca que exijam conduta imediata ou que permitam realização da corticoterapia prévia à resolução.

\section{Artéria cerebral média}

A avaliação da circulação cerebral fetal é realizada principalmente pela doplervelocimetria da artéria cerebral média. Outros vasos já foram estudados, mas a facilidade técnica na insonação deste vaso praticamente consagrou seu uso na prática obstétrica.

$\mathrm{Na}$ resposta fetal à hipóxia, enquanto o processo de centralização da circulação é eficiente para a manutenção da normoxia fetal, as atividades biofísicas permanecem preservadas. No agravamento do quadro, o feto apresentará alterações em suas atividades biofísicas, levando à anoxia, acidose e sofrimento fetal. Entretanto, estudos relatam resultados contraditórios sobre a aplicação clínica deste método. A diminuição do IP da artéria cerebral média é sinal de centralização na hipoxemia crônica, mas não é constatada associação com a gasometria de cordão no nascimento, indicando que o mecanismo compensatório é eficiente em manter a oxigenação cerebral por longo tempo $^{68}$. A perda da centralização antes do óbito fetal ${ }^{69,70}$ é relatada por alguns autores, mas não por outros ${ }^{71}$. E, ainda, fetos com restrição de crescimento apresentam diferentes padrões do IP da artéria cerebral média, e seu comportamento não é possível de ser generalizado para todos os $\operatorname{casos}^{72}$.

Ocorre certa variabilidade nas velocidades de fluxo deste vaso, portanto, recomenda-se avaliar, pelo menos, três medidas em três conjuntos de ondas na ausência de movimentos corpóreos ou respiratórios fetais. Segundo Mari $^{73}$, uma estratégia para a avaliação da circulação cerebral fetal é acessar os vasos em diferentes ocasiões durante o exame, em intervalos de cinco a dez minutos. Cada análise deve incluir pelo menos 10 a 15 ondas e caso exista variação nos resultados, a conclusão final deve se basear na média dos dados obtidos.

A doplervelocimetria da artéria cerebral média tem desempenhado papel fundamental no diagnóstico da anemia fetal, pela avaliação do pico de velocidade sistólica máxima ${ }^{74,75}$. Neste caso, a amostra é obtida na região proximal da artéria cerebral média, logo após sua origem na artéria carótida interna, e o ângulo de insonação recomendado é o menor possível, próximo a zero. A correção do ângulo utilizando o próprio aparelho de ultrassonografia pode ser aplicada para ângulos com diferença inferior a $30^{076}$. Entretanto, a correção inapropriada do ângulo aumenta a variabilidade interobservador ${ }^{77}$. Mesmo com todos os cuidados na técnica, uma taxa de erros entre 5 e $10 \%$ é observada na investigação da anemia fetal.

Em fetos com restrição de crescimento, a velocidade máxima elevada mostra ser melhor parâmetro para predição de mortalidade perinatal, quando comparado ao IP ${ }^{78}$. $\mathrm{Na}$ avaliação longitudinal destes índices, a velocidade máxima parece ser mais consistente, pois os seus valores aumentam conforme progride a idade gestacional, com discreta tendência à diminuição no período que antecede ao parto ou ao óbito fetal. Apesar desta tendência à diminuição, os valores da velocidade máxima continuam acima do limite superior da referência.

Nas últimas semanas de gestação, observa-se habitualmente um aumento da velocidade diastólica nas artérias cerebrais sem que isso represente sinal de comprometimento da oxigenação fetal ou centralização da circulação. Em fetos com resultados normais na doplervelocimetria da artéria umbilical, a avaliação da circulação cerebral não demonstrou associação com resultados perinatais adversos, indicando pouca utilidade do método em discriminar casos patológicos ${ }^{79}$. Portanto, as alterações na circulação cerebrais somente são valorizadas quando persistentes e interpretadas conforme a tendência de evolução de cada caso.

\section{Ducto venoso}

O ducto venoso comunica a veia umbilical com a veia cava inferior, possibilitando que o sangue proveniente do 
território placentário chegue ao átrio direito fetal e, assim, transpasse o forame oval e atinja órgãos nobres do feto, como o cérebro e o coração. Esta primeira intercomunicação é de grande importância, pois permite que o sangue com maior saturação de $\mathrm{O}_{2}$ seja direcionado diretamente ao coração fetal ${ }^{80}$.

A avaliação do fluxo no ducto venoso é realizada no plano transverso ou longitudinal do abdome superior fetal. Este vaso apresenta forma de trombeta, e o mapeamento colorido auxilia na identificação do seu istmo, local em que se verifica elevada velocidade de fluxo e turbilhonamento do sangue. Pela análise qualitativa do sonograma do ducto venoso, a ausência de fluxo diastólico na contração atrial, ou a onda 'a' reversa é sempre resultado que caracteriza anormalidade ${ }^{81}$ (Figura 1). A avaliação semiquantitativa é efetuada pelo IP para veias (IPV), que consiste na razão da diferença entre o pico de velocidade sistólico e a velocidade na contração atrial com a média da velocidade máxima da onda, e reflete a função cardíaca fetal ${ }^{82,83}$.

Várias anormalidades podem causar alterações $n$ doplervelocimetria do ducto venoso: redução da contratilidade cardíaca, aumento acentuado da pós-carga e arritmias. Essas alterações podem ser encontradas em fetos de gestações que cursam com insuficiência placentária na síndrome de transfusão feto-fetal, em fetos hidrópicos ou com arritmias.
$\mathrm{Na}$ insuficiência placentária, principalmente nos casos de manifestação precoce da restrição do crescimento fetal, a doplervelocimetria do ducto venoso tem particular importância ${ }^{84,85}$, pois verifica-se significativo aumento do fluxo para esse vaso com dilatação do diâmetro do seu istmo, favorecendo o aporte de sangue proveniente da veia umbilical para o coração e cérebro ${ }^{86}$. Em gestações com menos de 32 semanas que cursam com restrição do crescimento fetal, a mortalidade neonatal é significativamente maior quando a resolução da gestação é realizada nas fases de descompensação da circulação fetal, ou o ducto apresenta onda 'a' ausente ou reversa. A porcentagem de sobrevida, sem sequelas, entre os recém-nascidos é maior naqueles que apresentam, antes do nascimento, ducto venoso com onda 'a' com fluxo positivo ${ }^{87}$. Além disso, níveis elevados da troponina $T$ cardíaca fetal no nascimento associam-se ao aumento do IPV no ducto venoso, indicando disfunção do miocárdio fetal ${ }^{88}$.

Em estudo que analisa a sequência de anormalidades da insuficiência placentária, é possível verificar que ocorre aumento dos índices do ducto venoso cerca de cinco a oito dias antes da deterioração maior (onda 'a' ausente ou reversa), indicando a resolução da gravidez ${ }^{89}$. Portanto, para ser possível a conduta expectante em casos de insuficiência placentária grave, é necessária a vigilância estrita da vitalidade fetal, com base nos resultados do IPV do ducto venoso ${ }^{90}$, os quais indicam

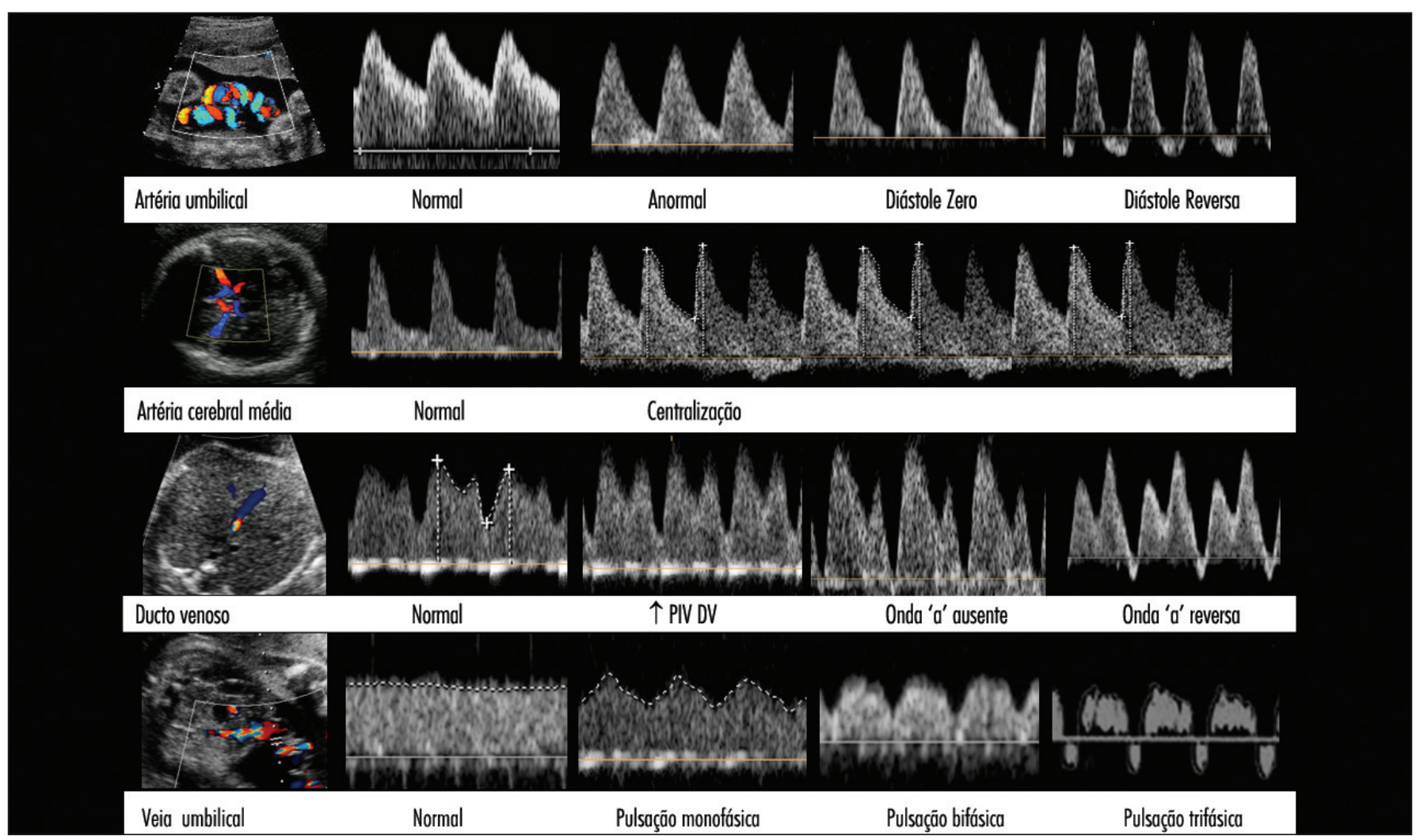

Figura 1 - Padrões dos sonogramas na dopplervelocimetria da artéria umbilical, artéria cerebral média, ducto venoso e veia umbilical. 
o comprometimento cardíaco fetal, bem como dos parâmetros biofísicos fetais que se alteram frente à acidemia fetal. Entretanto, essa vigilância é complexa, pois sabe-se que as manifestações cardiovasculares (doplervelocimetria do ducto venoso) e comportamentais (atividades biofísicas) da deterioração da condição fetal nos casos de restrição de crescimento podem ocorrer de forma independente, resultando em achados discordantes da doplervelocimetria em relação ao $\mathrm{PBF}^{91}$.

$\mathrm{Na}$ Clínica Obstétrica do Hospital das Clínicas da Faculdade de Medicina da Universidade de São Paulo (HCFMUSP), para os casos de restrição de crescimento fetal por insuficiência placentária grave, antes da $34^{\mathrm{a}}$ semana de gestação, é indicada a resolução mediata da gestação após um ciclo de corticoterapia quando o IPV do ducto venoso apresenta resultado entre 1,0 e 1,5. Caso o IPV apresente valor superior a 1,5 , a gravidez deve ser interrompida imediatamente ${ }^{92}$ pela descompensação cardíaca e circulatória fetal. Após a $34^{a}$ semana, as alterações dos exames de doplervelocimetria não são tão marcantes, de forma que a cardiotocografia e o PBF passam a desempenhar papel de maior importância na vigilância do bem-estar fetal.

\section{Veia umbilical}

O fluxo sanguíneo na veia umbilical é contínuo nas gestações normais após a $15^{\text {a }}$ semana de gestação. Antes da décima semana, as pulsações estão quase sempre presentes e o fluxo contínuo se estabelece progressivamente, passando a ser contínuo a partir da $12^{a}$ semana $^{93}$. Na restrição de crescimento fetal, a presença de pulsações pode estar associada à disfunção cardíaca.

A técnica frequentemente recomendada para a investigação do fluxo na veia umbilical refere-se ao exame do vaso em alça livre de cordão. É preconizada a análise qualitativa da morfologia do fluxo: com ou sem pulsações. Pulsações monofásicas podem ser observadas em até $20 \%$ dos fetos normais no terceiro trimestre ${ }^{94}$. Este achado é considerado benigno na ausência de outras alterações indicativas de comprometimento circulatório fetal.

$\mathrm{Na}$ avaliação qualitativa do fluxo da veia umbilical, as pulsações bifásicas ou trifásicas na veia umbilical são sinais indicativos de anormalidade (Figura 1). Na descompensação cardíaca fetal, a elevada pressão em átrio direito pode ser transmitida retrogradamente até a veia umbilical, resultando nos padrões de pulsação bifásica ou trifásica ${ }^{95}$. Nesses casos é comum observar importante anormalidade no fluxo do ducto venoso, com a onda 'a' ausente ou reversa. Em fetos hidrópicos, as pulsações na veia umbilical são indicativas de grave comprometimento do concepto, evoluindo para óbito em mais de $70 \%$ dos $\operatorname{casos}^{96}$.

\section{Indicações clínicas}

Serão abordadas as principais indicações clínicas para avaliação da vitalidade fetal.

\section{Síndromes hipertensivas}

A hipertensão arterial materna é a indicação mais comum, pois constitui importante fator de risco para comprometimento da vitalidade fetal. Os dados da literatura são insuficientes para determinar qual melhor teste a ser utilizado, quando iniciar ou com que frequência repetir os exames de vitalidade fetal. Não existem dados conclusivos indicando qual método teria melhor desempenho na avaliação antenatal de gestantes portadoras de pré-eclâmpsia ${ }^{97}$. Entretanto, nas gestações longe do termo, a partir da viabilidade fetal, a avaliação semanal pela cardiotocografia anteparto e o PBF são as condutas habitualmente recomendadas ${ }^{98,99}$. Nas situações em que há redução do volume de líquido amniótico, preconiza-se avaliação duas vezes na semana. Nos casos de restrição do crescimento fetal, a doplervelocimetria é de particular utilidade, notadamente quando existe aumento da resistência na placenta com índices anormais na artéria umbilical ${ }^{100}$. Antes da $34^{a}$ semana, a doplervelocimetria do ducto venoso norteará a conduta obstétrica ${ }^{92}$. Após esta idade gestacional, a cardiotocografia e o PBF passam a desempenhar papel de maior relevância, e as alterações nesses métodos serão de maior aplicabilidade na prática obstétrica.

\section{Restrição do crescimento fetal}

A restrição de crescimento intrauterino é fator de risco conhecido para o óbito perinatal. As anormalidades na doplervelocimetria das artérias umbilicais auxiliam a distinguir a restrição de crescimento fetal verdadeira, caracterizada pelos achados anormais na artéria umbilical, dos casos em que o feto é constitucionalmente pequeno, os quais apresentam valores normais. Não existem dados de estudos aleatorizados que demonstrem a melhor forma de avaliação fetal. Entretanto, a doplervelocimetria se destaca ao possibilitar acessar a hemodinâmica fetal nas diversas fases da resposta à hipóxia, melhorando o manejo dessas gestações ${ }^{101,102}$.

A ausência de fluxo diastólico ou reverso na artéria umbilical caracteriza quadro de insuficiência placentária grave que, na grande maioria dos casos, cursa com restrição do crescimento fetal. Nessas situações, é preconizada a avaliação diária da vitalidade fetal pelo elevado risco de comprometimento do concepto. O momento da resolução da gestação deve ponderar os riscos da prematuridade iatrogênica e do sofrimento fetal.

Os fetos classificados como pequenos constitucionais, sem alteração nos testes de vitalidade fetal, são 
acompanhados semanalmente e a resolução da gestação é programada para o termo ${ }^{92}$.

\section{Diabetes}

No passado, era elevada a mortalidade dos fetos das gestantes diabéticas insulino-dependentes. Porém, atualmente, com o aperfeiçoamento da terapêutica materna e das técnicas de monitorização fetal, a taxa de natimortos é equivalente à observada em gestações não complicadas. A mortalidade perinatal associa-se às anomalias congênitas, que devem ser detectadas precocemente entre a $18^{\mathrm{a}}$ e a $24^{\mathrm{a}}$ semana, pela ultrassonografia morfológica e ecocardiografia fetal. Estudos observacionais têm descrito a cardiotocografia anteparto de repouso e o PBF como métodos de avaliação da vitalidade fetal para as gestantes diabéticas ${ }^{103,104}$. Entretanto, nenhuma evidência demonstra qual método teria melhor desempenho no seguimento dessas gestações. De forma semelhante, não existem evidências que subsidiem a necessidade da avaliação rotineira da vitalidade fetal nos casos de diabetes gestacional controlada com dieta ${ }^{105}$.

A hiperglicemia materna levando à fetal pode promover aumento no vLA em diferentes semanas gestacionais, favorecendo o crescimento excessivo fetal ${ }^{106}$. No entanto, resultados controversos são constatados no seguimento de gestantes com diabetes pré-gestacional ${ }^{107}$. Após períodos de hiperglicemia materna e fetal, mesmo quando se obtém controle metabólico materno, nem sempre ocorre o retorno do vLA aos níveis normais. A excessiva movimentação respiratória fetal nos fetos de mães diabéticas também dificulta a correta interpretação dos exames ${ }^{108}$.

Nos casos de diabetes mellitus pré-gestacional, do tipo 1 , pode haver comprometimento do território placentário e a gestação pode cursar com insuficiência placentária, caracterizada por alteração precoce, a doplervelocimetria das artérias umbilicais e a restrição do crescimento fetal ${ }^{109}$. Estudos da morfometria placentária indicam que níveis glicêmicos maternos podem influenciar as características placentárias relacionadas com as trocas materno-fetais ${ }^{110}$.

\section{Gestação múltipla}

As gestações múltiplas são frequentemente associadas a fatores de risco que contribuem para maior morbidade perinatal como: idade materna avançada, prematuridade, pré-eclâmpsia, diabetes gestacional, crescimento fetal insuficiente, anormalidades na placentação, anomalias congênitas, entre outras. A determinação da corionicidade é fundamental para o seguimento, pois resultados adversos relacionam-se às gestações monocoriônicas ${ }^{111}$. Além disso, a morbidade perinatal precoce está aumentada em gêmeos com restrição de crescimento e discordância no peso ao nascimento superior a $20 \%{ }^{112}$.
Estudos específicos em gestações gemelares sugerem a avaliação semanal pela cardiotocografia, PBF e doplervelocimetria, como métodos isolados ou em combinação, para a predição de resultados adversos ${ }^{113}$. Não existem evidências suficientes para recomendar a idade gestacional, a partir de qual devem ser iniciados os testes para avaliação da vitalidade fetal. Também não existem recomendações específicas que suportem a avaliação antenatal de gestações múltiplas de ordem maior.

\section{Alteração do volume de líquido amniótico}

O oligohidrâmnio (ILA inferior a $5,0 \mathrm{~cm}$ ou diâmetro vertical do maior bolsão inferior a $2,0 \mathrm{~cm}$ ) e o polidrâmnio (ILA superior a $25,0 \mathrm{~cm}$ ou diâmetro vertical do maior bolsão superior a $8,0 \mathrm{~cm}$ ) são condições que frequentemente acompanhadas de outras morbidades maternas ou fetais: anomalias congênitas ${ }^{114}$, diabetes, hipertensão, rotura prematura de membranas, pós-datismo e restrição de crescimento fetal. Os critérios que definem os diagnósticos de oligohidrâmnio e polidrâmnio são controversos ${ }^{115,116}$, e recentemente têm sido questionados quanto à aplicação nas condutas da prática clínica. Em revisão sistemática, comparando métodos de avaliação do volume de líquido amniótico, não são encontradas evidências que demonstrem superioridade de um método em relação ao outro na prevenção de resultados adversos perinatais e é recomendada a utilização do critério de maior bolsão ${ }^{117}$.

No termo, o diagnóstico isolado de oligohidrâmnio ou polidrâmnio, sem outras complicações maternas ou fetais, nem sempre está associado a resultado adverso ${ }^{118,119}$. Entretanto, em gestações de alto risco, o oligohidrâmnio grave com ILA inferior a $3,0 \mathrm{~cm}$ associa-se a resultados de maior morbidade ${ }^{120}$.

\section{Pós-datismo}

O pós-datismo associa-se a maior morbidade e mortalidade perinatal. Recomenda-se que, ao completar 40 semanas, seja iniciada a monitoração da vitalidade fetal pela cardiotocografia e perfil biofísico fetal ${ }^{98}$. As complicações mais frequentemente observadas são o oligohidrâmnio e o mecônio no líquido amniótico, portanto recomenda-se a avaliação do vLA a cada três a quatro dias. A doplervelocimetria não se mostra útil para o seguimento das gestações com pós-datismo, pois é fraca a correlação dos índices na artéria umbilical com os resultados perinatais ${ }^{121}$.

\section{Diminuição da movimentação fetal}

A diminuição na movimentação pode ser sinal indicativo de comprometimento da vitalidade fetal. Está associada: a anomalias congênitas, à restrição de crescimento fetal, à prematuridade e ao óbito perinatal. Portanto, ela caracteriza situação em que é indicada a avaliação da 
vitalidade fetal, no mínimo, pela cardiotocografia ${ }^{122}$, ou em associação com a avaliação do vLA pela ultrassonografia e outros métodos ${ }^{123}$. Não existem evidências claras sobre a necessidade de avaliações posteriores quando os exames resultam normais.

\section{Considerações finais}

A literatura disponível sobre a aplicação dos testes de vitalidade fetal na prática obstétrica não demonstra evidência de protocolos ideais para o seguimento das gestações de risco. Muitos estudos são observacionais e as recomendações são baseadas em opiniões de especialistas. Existe clara necessidade da realização de estudos aleatorizados e controlados nesta área. Entretanto, é grande o desafio, tendo em vista a ampla variedade de condições anormais e fatores de confusão envolvidos. Novas pesquisas devem ser conduzidas para esclarecimento de pontos controvertidos como: idade gestacional apropriada para início da avaliação fetal em patologias específicas; intervalo de realização dos testes; e interpretação em gestações precoces, anteriores à $32^{a}$ semana gestacional.

\section{Referências}

1. Ministério da Saúde. Secretaria de Atenção à Saúde. Departamento de Ações Programáticas Estratégicas. Área Técnica de Saúde da Mulher. Pré-natal e puerpério: atenção qualificada e humanizada: manual técnico. Brasília: Ministério da Saúde; 2006

2. Grant A, Elbourne D, Valentin L, Alexander S. Routine formal fetal movement counting and risk of antepartum late death in normally formed singletons. Lancet. 1989;2(8659):345-9.

3. Mangesi L, Hofmeyr GJ. Fetal movement counting for assessment of fetal wellbeing. Cochrane Database Syst Rev. 2007; (1):CD004909.

4. Tveit JV, Saastad E, Stray-Pedersen B, Bordahl PE, Flenady V, Fretts $R$, et al. Reduction of late stillbirth with the introduction of fetal movement information and guidelines - a clinical quality improvement. BMC Pregnancy Childbirth. 2009;9:32.

5. Pattison N, McCowan L. Cardiotocography for antepartum fetal assessment. Cochrane Database Syst Rev. 2000;(2):CD001068.

6. Macones GA, Hankins GD, Spong CY, Hauth J, Moore T. The 2008 National Institute of Child Health and Human Development workshop report on electronic fetal monitoring: update on definitions, interpretation, and research guidelines. Obstet Gynecol. 2008;112(3):661-6.

7. ACOG practice bulletin. Antepartum fetal surveillance. Number 9, October 1999 (replaces Technical Bulletin Number 188, January 1994). Clinical management guidelines for obstetriciangynecologists. Int J Gynaecol Obstet. 2000;68(2):175-85

8. Electronic fetal heart rate monitoring: research guidelines for interpretation. National Institute of Child Health and Human Development Research Planning Workshop. Am J Obstet Gynecol. $1997 ; 177(6): 1385-90$

9. Pillai $M$, James $D$. The development of fetal heart rate patterns during normal pregnancy. Obstet Gynecol. 1990;76(5 Pt 1):812-6.

10. Westgate JA, Wibbens B, Bennet L, Wassink G, Parer JT, Gunn AJ. The intrapartum deceleration in center stage: a physiologic approach to the interpretation of fetal heart rate changes in labor. Am J Obstet Gynecol. 2007; 197(3):236.e 1-1 1.

11. Nomura RMY, Francisco RPV, Miyadahira S, Zugaib M. Cardiotocografia em gestações com diástole zero ou reversa nas artérias umbilicais: análise dos resultados perinatais. Rev Assoc Med Bras. 2003;49(1):79-85.
12. Nomura RMY, Francisco RPV, Miyadahira S, Zugaib M. Modelo matemático para predição da acidose metabólica no nascimento em gestações com diástole zero ou reversa. Rev Bras Ginecol Obstet. 2002;24(4):261-9.

13. Behle I, Zugaib M. Cardiotocografia anteparto de repouso. I. Considerações sobre conceito, metodologia e interpretação. Proposição de índice cardiotocométrico. Rev Bras Ginecol Obstet. $1981 ; 3(2): 72-85$

14. Tan KH, Sabapathy A. Fetal manipulation for facilitating tests of fetal wellbeing. Cochrane Database Syst Rev. $2001 ;(4)$ :CD003396.

15. Piyamongkol $W$, Trungtawatchai $S$, Chanprapaph $P$, Tongsong $T$. Comparison of the manual stimulation test and the nonstress test: a randomized controlled trial. J Med Assoc Thai. 2006;89(12): 1999-2002.

16. Tan $\mathrm{KH}$, Smyth R. Fetal vibroacoustic stimulation for facilitation of tests of fetal wellbeing. Cochrane Database Syst Rev. 2001;(1):CD002963.

17. Figueras F, Martínez JM, Puerto B, Coll O, Cararach V, Vanrell JA. Contraction stress test versus ductus venosus Doppler evaluation for the prediction of adverse perinatal outcome in growth-restricted fetuses with non-reassuring non-stress test. Ultrasound Obstet Gynecol. 2003;21(3):250-5

18. Freeman RK, Anderson G, Dorchester W. A prospective multiinstitutional study of antepartum fetal heart rate monitoring. II. Contraction stress test versus nonstress test for primary surveillance. Am J Obstet Gynecol. 1982;143(7):778-81

19. Silverman F, Suidan J, Wasserman J, Antoine C, Young BK. The Apgar score: is it enough? Obstet Gynecol. 1985;66(3):331-6.

20. Ayres-de-Campos D, Costa-Santos C, Bernardes J; SisPorto Multicentre Validation Study Group. Prediction of neonatal state by computer analysis of fetal heart rate tracings: the antepartum arm of the SisPorto multicentre validation study. Eur J Obstet Gynecol Reprod Biol. 2005; 1 18(1):52-60.

21. Dawes GS, Moulden M, Redman CW. System 8000: computerized antenatal FHR analysis. J Perinat Med. 1991;19(1-2):47-51.

22. Dawes GS, Moulden M, Redman CW. Improvements in computerized fetal heart rate analysis antepartum. J Perinat Med. 1996;24(1): 25-36. 
23. Serra V, Bellver J, Moulden M, Redman CW. Computerized analysis of normal fetal heart rate pattern throughout gestation. Ultrasound Obstet Gynecol. 2009;34(1):74-9.

24. Valensise H, Facchinetti F, Vasapollo B, Giannini F, Monte ID, Arduini $D$. The computerized fetal heart rate analysis in post-term pregnancy identifies patients at risk for fetal distress in labour. Eur J Obstet Gynecol Reprod Biol. 2006; 125(2): 185-92.

25. Galazios G, Tripsianis G, Tsikouras P, Koutlaki N, Liberis V. Fetal distress evaluation using and analyzing the variables of antepartum computerized cardiotocography. Arch Gynecol Obstet. In press; 2009.

26. Nomura RMY, Francisco RPV, Steinman DS, Miyadahira S, Zugaib M. Análise computadorizada da cardiotocografia anteparto em gestações de alto risco. Rev Bras Ginecol Obstet. 2002;24(1): 29-36.

27. Costa VN, Nomura RM, Reynolds KS, Miyadahira S, Zugaib M. Effects of maternal glycemia on fetal heart rate in pregnancies complicated by pregestational diabetes mellitus. Eur J Obstet Gynecol Reprod Biol. 2009;143(1):14-7.

28. Manning FA. Fetal biophysical profile. Obstet Gynecol Clin North Am. 1999;26(4):557-77.

29. Yamamoto RM, Francisco RPV, Miyadahira S, Zugaib M. Perfil biofísico fetal modificado: aspectos atuais na padronização do método. Rev Ginecol Obstet. 2000;1 1 (2): 1 18-22.

30. Magann EF, Doherty DA, Field K, Chauhan SP, Muffley PE, Morrison JC. Biophysical profile with amniotic fluid volume assessments. Obstet Gynecol. 2004; 104(1):5-10.

31. Manning FA. Dynamic ultrasound-based fetal assessment: the fetal biophysical profile score. Clin Obstet Gynecol. 1995;38(1): 26-44.

32. Francisco RPV, Yamamoto RM, Miyadahira S, Cunha CL, Zugaib M. Correlação entre testes para avaliação da vitalidade fetal, $\mathrm{pH}$ da artéria umbilical e os resultados neonatais em gestações de alto risco. Rev Bras Ginecol Obstet. 2000;22(8): 503-10.

33. Manning FA, Harman CR, Morrison I, Menticoglou SM, Lange IR, Johnson JM. Fetal assessment based on fetal biophysical profile scoring. IV. An analysis of perinatal morbidity and mortality. Am J Obstet Gynecol. 1990; 162(3):703-9.

34. Manning FA, Bondaji N, Harman CR, Casiro O, Menticoglou $\mathrm{S}$, Morrison I, et al. Fetal assessment based on fetal biophysical profile scoring. VIII. The incidence of cerebral palsy in tested and untested perinates. Am J Obstet Gynecol. 1998;178(4): 696-706.

35. Manning FA. Fetal biophysical profile: a critical appraisal. Clin Obstet Gynecol. 2002;45(4):975-85.

36. Manning FA, Morrison I, Harman CR, Lange IR, Menticoglou S. Fetal assessment based on fetal biophysical profile scoring: experience in 19,221 referred high-risk pregnancies. II. An analysis of false-negative fetal deaths. Am J Obstet Gynecol. 1987;157 (4 Pt 1):880-4

37. Dayal AK, Manning FA, Berck DJ, Mussalli GM, Avila C, Harman $C R$, et al. Fetal death after normal biophysical profile score: An eighteen-year experience. Am J Obstet Gynecol. 1999;181 (5 Pt 1):1231-6.

38. Nageotte MP, Towers CV, Asrat T, Freeman RK, Dorchester W. The value of a negative antepartum test: contraction stress test and modified biophysical profile. Obstet Gynecol. 1994;84(2):231-4.

39. Del Valle GO, Joffe GM, Izquierdo LA, Smith JF, Gilson GJ, Curet LB. The biophysical profile and the nonstress test: poor predictors of chorioamnionitis and fetal infection in prolonged preterm premature rupture of membranes. Obstet Gynecol. 1992;80(1):106-10.
40. Ghidini A, Salafia CM, Kirn V, Doria V, Spong CY. Biophysical profile in predicting acute ascending infection in preterm rupture of membranes before 32 weeks. Obstet Gynecol. 2000;96(2):201-6.

41. Honest H, Bachmann LM, Sengupta R, Gupta JK, Kleijnen J, Khan KS. Accuracy of absence of fetal breathing movements in predicting preterm birth: a systematic review. Ultrasound Obstet Gynecol. 2004;24(1):94-100.

42. FitzGerald DE, Drumm JE. Non-invasive measurement of human fetal circulation using ultrasound: a new method. $\mathrm{Br}$ Med J. 1977;2(6100): 1450-1

43. Yarlagadda P, Willoughby L, Maulik D. Effect of fetal heart rate on umbilical arterial Doppler indices. J Ultrasound Med. 1989;8(4):215-8

44. Sonesson SE, Fouron JC, Drblik SP, Tawile C, Lessard M, Skoll $A$, et al. Reference values for Doppler velocimetric indices from the fetal and placental ends of the umbilical artery during normal pregnancy. J Clin Ultrasound. 1993;21(5):317-24.

45. van den Wijngaard JA, van Eyck J, Noordam M, Wladimiroff JW, van Strik R. The Doppler flow velocity waveform in the fetal internal carotid artery with respect to fetal behavioural states. A longitudinal study. Biol Neonate. 1988;53(5):274-8.

46. Papageorghiou AT, Yu CK, Cicero S, Bower S, Nicolaides KH. Secondtrimester uterine artery Doppler screening in unselected populations: a review. J Matern Fetal Neonatal Med. 2002;12(2):78-88.

47. Papageorghiou AT, Yu CK, Bindra R, Pandis G, Nicolaides $\mathrm{KH}$; Fetal Medicine Foundation Second Trimester Screening Group. Multicenter screening for pre-eclampsia and fetal growth restriction by transvaginal uterine artery Doppler at 23 weeks of gestation. Ultrasound Obstet Gynecol. 2001;18(5):44 1-9.

48. Giles WB, Trudinger BJ, Baird PJ. Fetal umbilical artery flow velocity waveforms and placental resistance: pathological correlation. $\mathrm{Br}$ J Obstet Gynaecol. 1985;92(1):31-8.

49. Kingdom J, Huppertz B, Seaward G, Kaufmann P. Development of the placental villous tree and its consequences for fetal growth. Eur J Obstet Gynecol Reprod Biol. 2000;92(1):35-43.

50. Morrow RJ, Adamson SL, Bull SB, Ritchie JW. Effect of placental embolization on the umbilical arterial velocity waveform in fetal sheep. Am J Obstet Gynecol. 1989; 161 (4):1055-60.

51. Baschat AA, Gembruch U, Harman CR. The sequence of changes in Doppler and biophysical parameters as severe fetal growth restriction worsens. Ultrasound Obstet Gynecol. 2001;18(6):571-7.

52. Williams KP, Farquharson DF, Bebbington $M$, Dansereau J, Galerneau F, Wilson RD, et al. Screening for fetal well-being in a high-risk pregnant population comparing the nonstress test with umbilical artery Doppler velocimetry: a randomized controlled clinical trial. Am J Obstet Gynecol. 2003;188(5):1366-71

53. Omtzigt AM, Reuwer PJ, Bruinse HW. A randomized controlled trial on the clinical value of umbilical Doppler velocimetry in antenatal care. Am J Obstet Gynecol. 1994;170(2):625-34.

54. McCowan LM, Harding JE, Roberts AB, Barker SE, Ford C, Stewart AW. A pilot randomized controlled trial of two regimens of fetal surveillance for small-for-gestational-age fetuses with normal results of umbilical artery doppler velocimetry. Am J Obstet Gynecol. 2000;182(1 Pt 1):81-6.

55. Neilson JP, Alfirevic Z. Doppler ultrasound for fetal assessment in high risk pregnancies. Cochrane Database Syst Rev. 2000;(2):CD000073

56. Gagnon R, Van den Hof M; Diagnostic Imaging Committee, Executive and Council of the Society of Obstetricians and Gynaecologists of Canada. The use of fetal Doppler in obstetrics. J Obstet Gynaecol Can. $2003 ; 25(7): 601-14$ 
57. Westergaard HB, Langhoff-Roos J, Lingman G, Marsál K, Kreiner S. A critical appraisal of the use of umbilical artery Doppler ultrasound in high-risk pregnancies: use of meta-analyses in evidence-based obstetrics. Ultrasound Obstet Gynecol. $2001 ; 17(6): 466-76$.

58. Bricker L, Neilson JP. Routine Doppler ultrasound in pregnancy. Cochrane Database Syst Rev. 2000;(2):CD001450.

59. Goffinet F, Paris-Llado J, Nisand I, Bréart G. Umbilical artery Doppler velocimetry in unselected and low risk pregnancies: a review of randomised controlled trials. Br J Obstet Gynaecol. 1997; 104(4):425-30.

60. Arduini D, Rizzo G. Normal values of Pulsatility Index from fetal vessels: a cross-sectional study on 1556 healthy fetuses. J Perinat Med. 1990; 18(3): 165-72.

61. Nicolaides KH, Bilardo CM, Soothill PW, Campbell S. Absence of end diastolic frequencies in umbilical artery: a sign of fetal hypoxia and acidosis. BM. 1988;297(6655):1026-7.

62. Yamamoto RM, Francisco RPV, Miyadahira S, Chuba CC, Zugaib $M$. Fatores prognósticos para o óbito perinatal em gestações com diástole zero ou reversa na dopplervelocimetria das artérias umbilicais. Rev Bras Ginecol Obstet. 2000;22(6):353-63.

63. Karsdorp VH, van Vugt JM, van Geijn HP, Kostense PJ, Arduini D, Montenegro $\mathrm{N}$, et al. Clinical significance of absent or reversed end diastolic velocity waveforms in umbilical artery. Lancet. 1994;344(8938):1664-8.

64. Yamamoto RM, Miyadahira S, Francisco RPV, Steinman DS, Aquino MA, Zugaib M. Conduta obstétrica na diástole zero ou reversa à dopplervelocimetria das artérias umbilicais. Rev Ginecol Obstet. 1999; 10(1):35-9.

65. Zugaib M. Avaliação da vitalidade fetal. In: Zugaib M, editor. Obstetrícia. Barueri: Manole; 2008. p. 288-303.

66. Magalhães JA, Fritsch $A$. Crescimento intra-uterino restrito. In: Freitas F, Martins-Costa SH, Ramos JGL, Magalhães JA, editores. Rotinas em obstetrícia. $5^{a}$ ed. Porto Alegre: Artmed; 2006. p. 115-31.

67. Baschat AA, Viscardi RM, Hussey-Gardner B. Infant neurodevelopment following fetal growth restriction: relationship with antepartum surveillance parameters. Ultrasound Obstet Gynecol. 2009;33(1): 44-50.

68. Cheema R, Dubiel M, Gudmundsson S. Signs of fetal brain sparing are not related to umbilical cord blood gases at birth. Early Hum Dev. 2009;85(7):467-70.

69. Nomura RMY, Francisco RPV, Luca ACB, Miyadahira S, Banduki Neto JD, Zugaib M. Descentralização da circulação fetal precedendo o óbito em caso de diástole reversa na artéria umbilical. Rev Ginecol Obstet. 2001;12(2):79-82.

70. Mari G, Wasserstrum N. Flow velocity waveforms of the fetal circulation preceding fetal death in a case of lupus anticoagulant. Am J Obstet Gynecol. 1991;164(3):776-8.

71. Fieni S, Gramellini D, Piantelli G. Lack of normalization of middle cerebral artery flow velocity prior to fetal death before the 30th week of gestation: a report of three cases. Ultrasound Obstet Gynecol. 2004;24(4):474-6.

72. Nomura RMY, Francisco RPV, Chuba CC, Sakamoto K, Miyadahira S, Zugaib M. Óbito fetal em gestações com diástole zero ou reversa na dopplervelocimetria das artérias umbilicais: análise do perfil hemodinâmico fetal. Rev Ginecol Obstet. $2001 ; 12(4): 181-6$

73. Mari G. Doppler ultrasonography in obstetrics: from the diagnosis of fetal anemia to the treatment of intrauterine growth-restricted fetuses. Am J Obstet Gynecol. 2009;200(6): 613.e1-9.
74. Mari G, Deter RL, Carpenter RL, Rahman F, Zimmerman R, Moise KJ $\mathrm{Jr}$, et al. Noninvasive diagnosis by Doppler ultrasonography of fetal anemia due to maternal red-cell alloimmunization. Collaborative Group for Doppler Assessment of the Blood Velocity in Anemic Fetuses. N Engl J Med. 2000;342(1):9-14.

75. Nishie EN, Brizot ML, Liao AW, Carvalho MH, Toma O, Zugaib M. A comparison between middle cerebral artery peak systolic velocity and amniotic fluid optical density at $450 \mathrm{~nm}$ in the prediction of fetal anemia. Am J Obstet Gynecol. 2003;188(1):214-9.

76. Ruma MS, Swartz AE, Kim E, Herring AH, Menard MK, Moise $\mathrm{KJ}$ Jr. Angle correction can be used to measure peak systolic velocity in the fetal middle cerebral artery. Am J Obstet Gynecol. 2009;200(4):397.el-3.

77. Thomas JT, Muller P, Baghurst P, Wilkinson C. Interobserver variability in the measurement of fetal middle cerebral artery peak systolic velocity in a tertiary fetal medicine unit. Ultrasound Obstet Gynecol. 2008;32(1):77-81.

78. Mari G, Hanif F, Kruger M, Cosmi E, Santolaya-Forgas J, Treadwell MC. Middle cerebral artery peak systolic velocity: a new Doppler parameter in the assessment of growth-restricted fetuses. Ultrasound Obstet Gynecol. 2007;29(3):310-6.

79. Nomura RMY, Francisco RPV, Sakamoto K, Miyadahira S, Zugaib M. Centralização da circulação fetal em gestações de alto risco: avaliação da vitalidade fetal e resultados perinatais. Rev Bras Ginecol Obstet. 2001;23(3):137-43.

80. Kiserud T, Acharya G. The fetal circulation. Prenat Diagn. 2004;24(13): 1049-59.

81. Baschat AA, Gembruch U, Weiner CP, Harman CR. Qualitative venous Doppler waveform analysis improves prediction of critical perinatal outcomes in premature growth-restricted fetuses. Ultrasound Obstet Gynecol. 2003;22(3):240-5.

82. Mäkikallio K, Vuolteenaho $O$, Jouppila $P$, Räsänen J. Ultrasonographic and biochemical markers of human fetal cardiac dysfunction in placental insufficiency. Circulation. 2002; 105(17):2058-63.

83. Crispi F, Hernandez-Andrade E, Pelsers MM, Plasencia W, Benavides-Serralde JA, Eixarch E, et al. Cardiac dysfunction and cell damage across clinical stages of severity in growth-restricted fetuses. Am J Obstet Gynecol. 2008;199(3):254.e 1-8.

84. Andrade JQ, Miyadahira S, Nomura RMY, Francisco RPV, Zugaib M. Dopplervelocimetria dos compartimentos arterial e venoso da circulação fetal e umbilical em gestação de alto-risco: análise dos resultados perinatais. Rev Bras Ginecol Obstet. 2002;24(3): $153-60$.

85. Kiserud T, Kessler J, Ebbing C, Rasmussen S. Ductus venosus shunting in growth-restricted fetuses and the effect of umbilical circulatory compromise. Ultrasound Obstet Gynecol. 2006;28(2): 143-9.

86. Bellotti M, Pennati G, De Gasperi C, Bozzo M, Battaglia FC, Ferrazzi E. Simultaneous measurements of umbilical venous, fetal hepatic, and ductus venosus blood flow in growth-restricted human fetuses. Am J Obstet Gynecol. 2004;190(5):1347-58.

87. Baschat $A A$, Cosmi E, Bilardo $C M$, Wolf $H$, Berg C, Rigano S, et al. Predictors of neonatal outcome in early-onset placental dysfunction. Obstet Gynecol. 2007; 109(2 Pt 1):253-61.

88. Nomura RM, Cabar FR, Costa VN, Miyadahira S, Zugaib M. Cardiac troponin T as a biochemical marker of cardiac dysfunction and ductus venosus Doppler velocimetry. Eur J Obstet Gynecol Reprod Biol. In Press; 2009.

89. Turan OM, Turan S, Gungor S, Berg C, Moyano D, Gembruch U, et al. Progression of Doppler abnormalities in intrauterine growth restriction. Ultrasound Obstet Gynecol. 2008;32(2):160-7. 
90. Baschat AA. Doppler application in the delivery timing of the preterm growth-restricted fetus: another step in the right direction. Ultrasound Obstet Gynecol. 2004;23(2): 111 1-8.

91. Baschat AA, Galan HL, Bhide A, Berg C, Kush ML, Oepkes D, et al. Doppler and biophysical assessment in growth restricted fetuses: distribution of test results. Ultrasound Obstet Gynecol. 2006;27(1):41-7.

92. Zugaib M. Restrição do crescimento fetal. In: Zugaib $M$, editor. Obstetrícia. Barueri: Manole; 2008. p. 630-44.

93. Rizzo $G$, Arduini D, Romanini C. Umbilical vein pulsations: a physiologic finding in early gestation. Am J Obstet Gynecol. 1992; 167(3):675-7.

94. van Splunder IP, Huisman TW, Stijnen T, Wladimiroff JW. Presence of pulsations and reproducibility of waveform recording in the umbilical and left portal vein in normal pregnancies. Ultrasound Obstet Gynecol. 1994;4(1):49-53.

95. Hecher K, Campbell S, Doyle P, Harrington K, Nicolaides K. Assessment of fetal compromise by Doppler ultrasound investigation of the fetal circulation. Arterial, intracardiac, and venous blood flow velocity studies. Circulation. 1995;91(1):129-38.

96. Tulzer G, Gudmundsson S, Wood DC, Cohen AW, Weiner S, Huhta JC. Doppler in non-immune hydrops fetalis. Ultrasound Obstet Gynecol. 1994;4(4):279-83.

97. Mulrow CD, Chiquette E, Ferrer RL, Sibai BM, Stevens KR, Harris $M$, et al. Management of chronic hypertension during pregnancy. Evid Rep Technol Assess (Summ). 2000;(14): 1-4.

98. Miyadahira S. Vitalidade fetal. In: Zugaib M, Bittar RE, editores. Protocolos assistenciais da clínica obstétrica FMUSP. 3a ed. São Paulo: Atheneu; 2007. p. 107-25.

99. [No authors listed]. Report of the National High Blood Pressure Education Program Working Group on High Blood Pressure in Pregnancy. Am J Obstet Gynecol. 2000; 183(1):S1-S22.

100. Miyadahira S, Yamamoto RM, Komagata HA, Kahhale S, Zugaib M. Dopplerfluxometria umbilical e uterina nas gestações complicadas pelas síndromes hipertensivas. Rev Ginecol Obstet. 1993;4(3): 128-36.

101. Sasaki S, Bittar RE, Martinelli S, Yamasaki AA, Miyadahira S, Nomura RMY, et al. Dopplervelocimetria arterial em gestantes com antecedente de crescimento intra-uterino retardado. Rev Bras Ginecol Obstet. 1998;20(9):517-24.

102. Miyadahira S, Nomura RMY, Zugaib M. Seqüência de alterações do Doppler na circulação fetal central e periférica. Rev Assoc Med Bras. 2003;49(3):228.

103. Kjos SL, Leung A, Henry OA, Victor MR, Paul RH, Medearis AL. Antepartum surveillance in diabetic pregnancies: predictors of fetal distress in labor. Am J Obstet Gynecol. 1995; 173(5):1532-9.

104. Nomura RMY, Costa VN, Sakamoto K, Maganha CA, Miyadahira S, Zugaib M. Cardiotocografia computadorizada em gestações complicadas pelo diabete melito pré-gestacional: padrões da freqüência cardíaca em fetos grandes para a idade gestacional. Rev Bras Ginecol Obstet. 2005;27(12):712-8.

105. Landon $M B$, Vickers $S$. Fetal surveillance in pregnancy complicated by diabetes mellitus: is it necessary? J Matern Fetal Neonatal Med. $2002 ; 12(6): 413-6$.

106. Nomura RMY, Francisco RPV, Maganha CA, Miyadahira S, Banduki Neto JD, Zugaib M. Vitalidade fetal em gestações complicadas com diabete melito pré-gestacional: um estudo longitudinal. Rev Bras Ginecol Obstet. 2002;24(2):1 13-20.

107. Maganha CA, Nomura RMY, Zugaib M. Associação entre perfil glicêmico materno e o índice de líquido amniótico em gestações complicadas pelo Diabetes mellitus pré-gestacional. Rev Assoc Med Bras. 2009;55(2): 169-74.

108. Nomura RMY, Martins AN, Teshima LK, Miyadahira S, Zugaib M. Movimentos respiratórios fetais em gestações com diabetes mellitus pré-gestacional. Rev Bras Ginecol Obstet. 2007;29(7):352-7.

109. Yamamoto RM, Francisco RPV, Miyadahira S, Banduki Neto JD, Zugaib M. Avaliação da vitalidade fetal em gestantes diabéticas: análise dos resultados neonatais. Rev Bras Ginecol Obstet. 2000;22(9):557-66.

1 10. Calderon IM, Damasceno DC, Amorin RL, Costa RA, Brasil MA, Rudge MV. Morphometric study of placental villi and vessels in women with mild hyperglycemia or gestational or overt diabetes. Diabetes Res Clin Pract. 2007;78(1):65-71.

111. Hack KE, Kaandorp JJ, Derks JB, Elias SG, Pistorius L, Visser GH. The value of umbilical artery Doppler velocimetry in the antenatal surveillance of uncomplicated monochorionic twin pregnancies. Ultrasound Obstet Gynecol. 2008;31(6):662-8.

112. Alam Machado Rde C, Brizot Mde L, Liao AW, Krebs VL, Zugaib M. Early neonatal morbidity and mortality in growth-discordant twins. Acta Obstet Gynecol Scand. 2009;88(2):167-71.

113. Yamamoto RM, Francisco RPV, Miyadahira S, Okatani D, Banduki Neto JD, Zugaib M. Avaliação da vitalidade fetal em gestações gemelares e análise dos resultados neonatais. Rev Ginecol Obstet. 2000; 11 (1):22-9.

114. Noronha Neto C, Souza ASR, Moraes Filho OB, Noronha AMB. Volume do líquido amniótico associado às anomalias fetais diagnosticadas em um centro de referência do nordeste brasileiro. Rev Bras Ginecol Obstet. 2009;31 (4): 164-70.

115. Magann EF, Chauhan SP, Doherty DA, Magann MI, Morrison JC. The evidence for abandoning the amniotic fluid index in favor of the single deepest pocket. Am J Perinatol. 2007;24(9):549-55.

116. Magann EF, Doherty DA, Chauhan SP, Busch FW, Mecacci F, Morrison JC. How well do the amniotic fluid index and single deepest pocket indices (below the 3rd and 5th and above the 95th and 97 th percentiles) predict oligohydramnios and hydramnios? Am J Obstet Gynecol. 2004;190(1):164-9.

117. Nabhan AF, Abdelmoula YA. Amniotic fluid index versus single deepest vertical pocket: a meta-analysis of randomized controlled trials. Int J Gynaecol Obstet. 2009;104(3):184-8.

1 18. Magann EF, Chauhan SP, Doherty DA, Lutgendorf MA, Magann $\mathrm{MI}$, Morrison JC. A review of idiopathic hydramnios and pregnancy outcomes. Obstet Gynecol Surv. 2007;62(12): 795-802.

119. Zhang J, Troendle J, Meikle S, Klebanoff MA, Rayburn WF. Isolated oligohydramnios is not associated with adverse perinatal outcomes. BJOG. 2004; 111 (3):220-5.

120. Nomura RMY, Francisco RPV, Miyadahira S, Zugaib M. Análise dos testes de vitalidade fetal e dos resultados perinatais em gestações de alto risco com oligoidrâmnio. Rev Bras Ginecol Obstet. 2002;24(6):401-6.

121. Guidetti DA, Divon MY, Cavalieri RL, Langer O, Merkatz IR. Fetal umbilical artery flow velocimetry in postdate pregnancies. Am J Obstet Gynecol. 1987;157(6):1521-3.

122. Olesen AG, Svare JA. Decreased fetal movements: background, assessment, and clinical management. Acta Obstet Gynecol Scand. 2004;83(9):818-26.

123. Haws RA, Yakoob MY, Soomro T, Menezes EV, Darmstadt GL, Bhutta ZA. Reducing stillbirths: screening and monitoring during pregnancy and labour. BMC Pregnancy Childbirth. 2009;9 Suppl 1:S5. 